\title{
Editorial
}

\section{Revisión crítica del uso de radiación como tratamiento inicial del cáncer de próstata localizado}

\author{
Critical review of use of radiation as initial treatment for localized
} prostate cancer

El perfil actual del paciente con cáncer de próstata (CaP) corresponde a un varón todavía "joven", con una larga expectativa de vida, sexualmente activo y sin problemas miccionales previos relevantes. En nuestro medio más del $75 \%$ de los casos de CaP se diagnostican en estadio localizado ${ }^{1}$. El principal objetivo en los pacientes con CaP localizado será realizar un tratamiento que ofrezca la mayor tasa de curación con la mínima morbilidad funcional (continencia urinaria y potencia sexual), pero la segunda prioridad se establecerá si falla el tratamiento inicial y se requiere de una terapia de rescate, donde el objetivo debería continuar siendo la intención curativa con el mínimo perjuicio funcional.

Múltiples trabajos respaldan que los tratamientos con más experiencia y eficacia son la prostatectomía radical (PR) y la radioterapia (RT), ya sea externa o braquiterapia ${ }^{1}$, y aunque son consideradas técnicas con resultados similares, hasta hoy no existe ningún estudio prospectivo y aleatorizado que compare ambos tratamientos. Un reciente trabajo de revisión basado en la base de datos CAPSURE (Cancer Prostate Strategic Urological Research Endevor) muestra que aproximadamente el $30 \%$ de hombres con CaP fracasan al tratamiento inicial, y de estos, el $63 \%$ había realizado RT y sólo el $23 \%$ se había sometido a $\mathrm{PR}^{2}$. Aunque un análisis detallado pone de manifiesto que el $19 \%$ de pacientes sometidos a PR y el $45 \%$ de irradiados presentaban tumores de alto riesgo, el resto de parámetros fueron completamente homogéneos entre ambos grupos de tratamiento ${ }^{2}$. Independientemente de los resultados de este estudio, y aunque hasta el momento no se ha demostrado que un tipo de tratamiento sea mejor que otro en cuanto a tasa de curación, es posible abrir una discusión sobre qué tratamiento es mejor, basada en la observación de diversos detalles referentes a eficacia, seguimiento, morbilidad y opciones de rescate ante un fracaso en el tratamiento del CaP localizado. Para ello se han elegido algunos trabajos aparecidos recientemente que cuestionan la supuesta equivalencia entre cirugía y radioterapia.

\section{Control oncológico}

El control oncológico de los pacientes sometidos a RT por CaP localizado se realiza con medición de los niveles plasmáticos de antígeno prostático específico (PSA) ${ }^{1}$. Los criterios de definición de recidiva bioquímica después de RT han ido cambiando en los últimos años, buscando un estándar de objetividad todavía pendiente de alcanzar. La primera definición fue establecida por un panel de consenso de la ASTRO (American Society Therapeutic Radiology and Oncology) en el año 1996, como tres elevaciones consecutivas de PSA a partir del valor nadir ${ }^{3}$. La experiencia demostró que esta definición era dependiente de la frecuencia y duración del seguimiento, y quedaba sustancialmente artefactada. Por esta razón, otro panel de consenso de la ASTRO en 2005 redefinió la recidiva bioquímica como el punto de corte numérico obtenido por la suma de un valor de PSA de $2 \mathrm{ng} /$ $\mathrm{ml}$ al valor nadir, obtenido después de la $\mathrm{RT}^{4}$, y que es el criterio válido en la actualidad.

Por su parte, el resultado de la PR está sujeto exclusivamente a la exéresis completa de la glándula prostática, y el control se centra en la búsqueda de un PSA indetectable. Desde los inicios de la técnica las diferentes asociaciones de Urología consensuaron que dos elevaciones consecutivas con un nivel de PSA $\geq 0,2 \mathrm{ng} / \mathrm{ml}$ según la European Association of Urology (EAU) y $>0,4 \mathrm{ng} / \mathrm{ml}$ según la American Urological Association (AUA), eran las definiciones estándar de recidiva bioquímica después de PR. Dada la ausencia de tejido prostático el uso del PSA post-PR se convierte en una herramienta muy sensible para detectar un fracaso de tratamiento.

En contraposición, el criterio de la ASTRO evidencia la dificultad de control oncológico post-RT mediante PSA, motivado por el prolongado e imprevisible efecto terapéutico de la radiación, así como la persistencia de tejido residual prostático funcionante productor de PSA. Estas circunstancias y la diferente sistemática para medir los resultados conllevan 
sesgos y limitaciones metodológicas inevitables cuando se realizan estudios que comparan tasas de control oncológico entre PR y RT.

Un reciente trabajo de Walsh et $\mathrm{al}^{5}$ analiza cómo serían los resultados oncológicos de la PR si se aplicara un criterio equivalente al aceptado en la actualidad por la ASTRO. El estudio permite observar una sobreestimación de la supervivencia libre de recidiva bioquímica, un retraso en la determinación de la recidiva y un aumento (prácticamente el triple) del tiempo medio libre de recidiva. Por todo esto, no parecería correcto comparar tasas de control oncológico después de PR y RT en el CaP localizado con los criterios recidiva después de RT vigentes en la actualidad. Además, otro reciente estudio ratifica las limitaciones del PSA para la monitorización del CaP tratado con $\mathrm{RT}^{6}$. Se analizan 21 pacientes tratados con RT por CaP que, durante su seguimiento, fueron sometidos a cistoprostatectomía radical (CPR) por causas no relacionadas con su neoplasia prostática, la mayoría (19 casos) por cáncer vesical infiltrante. Diecisiete pacientes habían sido tratados del CaP con RT externa y 4 con braquiterapia. Sólo 18 de los 21 pacientes tenían un PSA de control post-RT evaluable en el momento de la revisión, de los cuales 16 (89\%) cumplían criterios de estar libres de recidiva bioquímica según la ASTRO 2005. Sin embargo, el análisis anatomopatológico de las piezas de CPR demostró CaP residual en el 52\% (11 de 21 casos), es decir, más de la mitad de los pacientes que supuestamente estaban libres de recidiva según criterios de PSA-ASTRO 2005 presentaban CaP residual. Es bien conocido que el efecto curativo de la RT sobre un CaP puede demorarse hasta los 12-18 meses. Este es el motivo por el que no se recomienda realizar biopsias de próstata hasta 24-36 meses post-RT, evitando así falsos positivos ${ }^{7}$. Este estudio presentaba una duración media de tiempo entre la RT y la CPR de 60 meses, cuando la RT había realizado ya todo su efecto terapéutico ${ }^{8}$, poniendo en evidencia las limitaciones del PSA en el control del CaP tratado con RT, así como las dificultades de la RT para lograr un control inmediato o definitivo de la enfermedad. Además, terapias adyuvantes, como el bloqueo hormonal, que se siguen asociando habitualmente a la RT, condicionan la calidad de vida del paciente, encarecen el tratamiento $y$, sobre todo, dificultan todavía más la valoración de resultados. Frente a estos inconvenientes la valoración de los resultados después de la PR resulta sencilla y objetiva, siendo posible adoptar medidas terapéuticas inmediatas si se detecta la persistencia o recidiva de la enfermedad.

\section{Resultados funcionales}

En los casos tratados con PR en que se detecta enfermedad residual o un fracaso bioquímico local es posible recurrir a la RT como maniobra de rescate. Las terapias de rescate deben perseguir el mismo objetivo que el tratamiento inicial: garantizar al máximo los resultados oncológicos y funcionales. El control oncológico después de un tratamiento de rescate, sea con RT o con PR, ofrece resultados oncológicos similares ${ }^{8}$. Sin embargo, desde el punto de vista funcional, la morbilidad urinaria, sexual y digestiva es muy distinta según el tratamiento inicial $\mathrm{y}$ de rescate utilizados. Mientras que la RT es un recurso sencillo de aplicar tras una PR, la maniobra inversa (cirugía sobre RT previa) compromete mucho más la obtención de resultados satisfactorios para el paciente. Los trabajos que analizan el uso de RT adyuvante o de rescate después de PR observan mayores efectos adversos sexuales y rectales, sin empeoramientos no obstante del estado de continencia que se presentaba tras la $\mathrm{PR}^{8}$, incluso después de un rescate con $\mathrm{RT}$ de alta tasa $(70 \mathrm{~Gy})^{9}$. Cuando el tratamiento primario es RT y se opta por una PR de rescate la morbilidad se multiplica, incluso en manos expertas, con tasas de incontinencia superiores al 50\% ${ }^{10}$, aumentando además el riesgo de esclerosis cervical y la pérdida completa de potencia sexual en los pacientes que todavía la conservaban ${ }^{11}$. Además, existe un mayor riesgo de lesión rectal durante la $\mathrm{PR}$ en el territorio irradiado, que probablemente condenará al paciente a una colostomía definitiva. Incluso mediante abordajes laparoscópicos o robóticos, y aun siendo técnicamente factibles, se observa mayor morbilidad ${ }^{12}$. El problema se evidencia sobre todo en el perfil de paciente sano y con larga expectativa de vida, donde además de que la $P R$ de rescate aumentará severamente la morbilidad, el resto de alternativas locales de rescate existentes (crioterapia, HIFU [High Intensity Focused Ultrasround]) no tienen ni un seguimiento ni una evidencia científica suficiente para ser recomendadas, y están expuestas a importante morbilidad cuando se aplican como rescate post-RT. Esta situación condena a nuestro "joven" paciente con CaP localizado, tratado con RT y con recidiva local, a un tratamiento paliativo, como un bloqueo hormonal de por vida, que aunque pueda plantearse intermitente, asociará efectos adversos demostrados a medio y largo plazo ${ }^{13}$. Además, ante una situación de incontinencia y/o impotencia después de un tratamiento de un CaP localizado, la posibilidad de colocar un esfínter artificial y/o prótesis peneana será más factible en una zona que sólo ha sido sometida a cirugía que en un territorio irradiado, donde la cirugía posterior resulta muy compleja y con menores posibilidades de éxito.

\section{Efectos adversos y complicaciones}

El desarrollo de segundas neoplasias en pacientes tratados por CaP, con cierta predisposición a asentar en la vejiga, es otra cuestión a destacar ${ }^{14}$. Los factores más relacionados con este hecho son el tabaquismo y la RT previa sobre la zona pélvica ${ }^{14,15}$. Los pacientes sometidos a RT por CaP presentan aún mayor riesgo de desarrollar un segundo cáncer, tanto en la pelvis como en las zonas próximas al campo irradiado ${ }^{16}$. Estudios sobre una amplia población muestran que la RT prostática se asocia, de forma estadísticamente significativa, a un riesgo aumentado de desarrollar un segundo tumor, sobre todo en pacientes con una larga supervivencia ${ }^{17,18}$. Dicho mayor riesgo relativo se hace patente con el tiempo, alcanzando el 34\% de pacientes después de más de 10 años de la irradiación ${ }^{17}$. La localización 
más común es la vejiga y el recto, aunque también órganos alejados, incluso cuando se empleó IMRT (radioterapia de intensidad modulada) ${ }^{18}$. Estos segundos tumores suelen ser de mayor grado y con patrones histológicos más agresi$\operatorname{vos}^{19}$. También se han descrito segundos tumores después de braquiterapia ${ }^{20}$. Son los pacientes con CaP localizado y larga expectativa de vida los que se verán más afectados por este riesgo de segundas neoplasias después de RT. En contraste, no existe ningún estudio que demuestre alguna relación entre la PR y el desarrollo de segundas neoplasias en el tratamiento del CaP.

Actualmente se está produciendo un cambio en el esquema terapéutico de la RT externa, que evoluciona hacia modelos de conformación de campos y hacia la IMRT. Esta modalidad abarca más campos y usa mayores dosis en la zona del tumor, exponiendo mayores volúmenes de tejido normal a dosis bajas de radiación. Tanto la RT conformada como la IMRT presentan, además del riesgo aumentado de segundos tumores anteriormente mencionado, un mayor riesgo de complicaciones graves, que aunque poco frecuentes (cistopatía y rectitis rádicas, atrapamiento ureteral, dermatitis), en ocasiones precisarán de medidas agresivas, como la cistectomía paliativa o la derivación intestinal ${ }^{21}$.

La PR abierta se ha asociado desde su descripción a morbilidad relacionada con la continencia y la potencia, pero en los últimos años los avances en el conocimiento de la anatomía prostática, así como la espectacular evolución de la técnica quirúrgica, gracias a la laparoscopia y la robótica, han permitido obtener unos excelentes resultados oncológicos y funcionales ${ }^{22}$. Además, la cirugía se realiza con unas pérdidas hemáticas mínimas, siendo la estancia hospitalaria y el periodo con sonda de pocos días. El argumento clásico de que la RT aporta ventajas en cuanto a continencia y potencia, así como la evitación de los inconvenientes de la cirugía -herida quirúrgica, sonda vesical-, queda neutralizado por los últimos resultados de la PR laparoscópica y robótica ${ }^{22}$.

\section{Conclusión}

Todas estas cuestiones deben provocar en urólogos y radioterapeutas una reflexión crítica sobre la indicación del uso de la RT como tratamiento inicial en el CaP localizado. A partir de los argumentos comentados la PR debería contemplarse como la única opción inicial de tratamiento del CaP localizado en el frecuente perfil de paciente "joven", con una larga expectativa de vida, sexualmente activo y sin trastornos miccionales. A los pacientes a los que se ofrece de entrada una elección equivalente entre PR o RT se les debe informar en términos realistas de la eficacia y resultados de ambas técnicas, modalidad de seguimiento, efectos adversos a corto y largo plazo, así como las opciones de rescate ante un fracaso de una u otra terapia.

\section{B I B L I O G R A F Í A}

1. Bracarda S, de Cobelli O, Greco C, Prayer-Galetti T, Valdagni $\mathrm{R}$, Gatta G, et al. Cancer of the prostate. Crit Rev Oncol Hematol. 2005;56(3):379-96.
2. Agarwal PK, Sadetsky N, Konety BR, Resnick MI, Carroll PR; Cancer of the Prostate Strategic Urological Research Endeavor (CaPSURE). Treatment failure after primary and salvage therapy for prostate cancer: likelihood, patterns of care, and outcomes. Cancer. 2008;112(2):307-14.

3. American Society of Therapeutic Radiology and Oncology Consensus Panel: Consensus statement: guidelines for PSA following radiation therapy. Int J Radiat Oncol Biol Phys. 1997;37:1035-41.

4. Roach M 3rd, Hanks G, Thames H Jr, Schellhammer P, Shipley WU, Sokol GH, et al. Defining biochemical failure following radiotherapy with or without hormonal therapy in men with clinically localized prostate cancer: recommendations of the RTOG-ASTRO Phoenix Consensus Conference. Int J Radiat Oncol Biol Phys. 2006;65(4):965-74.

5. Nielsen ME, Makarov DV, Humphreys E, Mangold L, Partin AW, Walsh PC, et al. Is it possible to compare PSA recurrence-free survival after surgery and radiotherapy using revised ASTRO criterion--"nadir + 2"? Urology. 2008;72(2):389-93.

6. Kaplan DJ, Crispen PL, Greenberg RE, Chen DY, Viterbo R, Buyyounouski MK, et al. Residual prostate cancer after radiotherapy: a study of radical cystoprostatectomy specimens. Urology. 2008;72(3):654-8.

7. Crook J, Malone S, Perry G, Bahadur Y, Robertson S, Abdolell M. Postradiotherapy prostate biopsies: what do they really mean? Results for 498 patients. Int J Radiat Oncol Biol Phys. 2000;48(2):355-67.

8. Hu JC, Elkin EP, Krupski TL, Gore J, Litwin MS. The effect of postprostatectomy external beam radiotherapy on quality of life: results from the Cancer of the Prostate Strategic Urologic Research Endeavor. Cancer. 2006;107(2):281-8.

9. Jung C, Cookson MS, Chang SS, Smith JA Jr, Dietrich MS, Teng $\mathrm{M}$. Toxicity following high-dose salvage radiotherapy after radical prostatectomy. BJU Int. 2007;99(3):529-33.

10. Hautmann RE. Salvage radical prostatectomy. BJU Int. 2006; 45(10):1260-5.

11. Darras J, Joniau S, Van Poppel H. Salvage radical prostatectomy for radiorecurrent prostate cancer: indications and results. Eur J Surg Oncol. 2006.;32(9):964-9.

12. Kaouk JH, Hafron J, Goel R, Haber GP, Jones JS. Robotic salvage retropubic prostatectomy after radiation/brachytherapy: initial results. BJU Int. 2008;102(1):93-6.

13. Isbarn H, Boccon-Gibod L, Carroll PR, Montorsi F, Schulman C, Smith MR, et al. Androgen deprivation therapy for the treatment of prostate cancer: consider both benefits and risks. Eur Urol. 2009;55(1):62-75.

14. Kellen E, Zeegers MP, Dirx M, Houterman S, Droste J, Lawrence G, et al. Occurrence of both bladder and prostate cancer in five cancer registries in Belgium, The Netherlands and the United Kingdom. Eur J Cancer. 2007;43(11): 1694-700.

15. Neugut AI, Ahsan H, Robinson E, Ennis RD. Bladder carcinoma and other second malignancies after radiotherapy for prostate carcinoma. Cancer. 1997;79(8):1600-4.

16. Brenner DJ. Induced second cancers after prostate-cancer radiotherapy: no cause for concern? Int J Radiat Oncol Biol Phys. 2006;65(3):637-9.

17. Brenner DJ, Curtis RE, Hall EJ, Ron E. Second malignancies in prostate carcinoma patients after radiotherapy compared with surgery. Cancer. 2000;15;88(2):398-406.

18. Moon K, Stukenborg GJ, Keim J, Theodorescu D. Cancer incidence after localized therapy for prostate cancer. Cancer. 2006;107(5):991-8. 
19. Hall EJ, Wuu CS. Radiation-induced second cancers: the impact of 3D-CRT and IMRT. Int J Radiat Oncol Biol Phys. 2003;56 (1):83-8.

20. Liauw SL, Sylvester JE, Morris CG, Blasko JC, Grimm PD. Second malignancies after prostate brachytherapy: incidence of bladder and colorectal cancers in patients with 15 years of potential follow-up. Int J Radiat Oncol Biol Phys. 2006;66(3):669-73.

21. Loeb S, Nadler RB. Management of the complications of external beam radiotherapy and brachytherapy. Curr Urol Rep. 2006;7(3):200-8.

22. Ficarra V, Novara G, Artibani W, Cestari A, Galfano A, Graefen $\mathrm{M}$, et al. Retropubic, Laparoscopic, and Robot-Assisted
Radical Prostatectomy: A Systematic Review and Cumulative Analysis of Comparative Studies. Eur Urol. 2009;55(5):

1037-63.

S. Esquena*, F.M. Sánchez-Martín, J. Palou Redorta, A. Rosales Bordes, Ó. Rodríguez-Faba y H. Villavicencio Mavrich Servicio de Urología, Fundació Puiguert, Barcelona, España

*Autor para correspondencia. Correo electrónico: dr.esquena@gmail.com

(S. Esquena) 